DOI: 10.17516/1997-1397-2021-14-2-133-143

УДК 517.956

\title{
Solvability of BVPs for the Parabolic-Hyperbolic Equation with Non-linear Loaded Term
}

\author{
Obidjon Kh. Abdullaev* \\ Romanovsky Institute of Mathematics \\ Tashkent, Uzbekistan \\ National University of Uzbekistan \\ Tashkent, Uzbekistan
}

Received 10.09.2020, received in revised form 10.11.2020, accepted 20.02.2021

\begin{abstract}
This work is devoted to prove the existence and uniqueness of solution of BVP with non-local assumptions on the boundary and integral gluing conditions for the parabolic-hyperbolic type equation involving Caputo derivatives. Using the method of integral energy, the uniqueness of solution have been proved. Existence of solution was proved by the method of integral equations.
\end{abstract}

Keywords: Caputo fractional derivatives, loaded equation, integral gluing condition, non-linear integral equation, non-local problem, existence and uniqueness of solution.

Citation: O.Kh. Abdullaev, Solvability of BVPs for the Parabolic-hyperbolic Equation with Non-linear Loaded Term, J. Sib. Fed. Univ. Math. Phys., 2021, 14(2), 133-143.

DOI: 10.17516/1997-1397-2021-14-2-133-143.

Boundary value problem (BVP) for the mixed type equation of fractional order is one of the intensively developing lines of study in the field of partial differential equations. Local and nonlocal problems for the parabolic-hyperbolic type equations involving several integro-differential operators of fractional order was investigated by many authors (see [1-3] and references therein).

BVPs for loaded partial differential equations arise in problems of optimal control of agroeconomic systems, for example, in the problem of controlling the label of ground waters and soil moisture (see $[4,5]$ and references therein). Some results in the theory of BVPs for the loaded equations of parabolic, parabolic-hyperbolic and elliptic-hyperbolic types were presented in [6-8]. Integral boundary conditions have various applications in thermo-elasticity, chemical engineering, population dynamics, etc. Integral gluing conditions were used in $[9,10]$ and in related works.

In this paper we consider the following parabolic-hyperbolic type equation of fractional order with non-linear loaded term:

$$
0=\left\{\begin{array}{ll}
u_{x x}-{ }_{C} D_{o y}^{\alpha} u+f_{1}(x, y ; u(x, 0)) & \text { at } y>0 \\
u_{x x}-u_{y y}+f_{2}(x, y ; u(x, 0)) & \text { at } y<0
\end{array},\right.
$$

where ${ }_{C} D_{\text {oy }}^{\alpha} u$ is the Caputo derivative with fractional order $\alpha(0<\alpha<1)$ defined as (see [10], p. 92)

$$
\left({ }_{C} D_{a y}^{\alpha} f\right) y=\frac{1}{\Gamma(1-\alpha)} \int_{a}^{y} \frac{f^{\prime}(t)}{(y-t)^{\alpha}} d t, \quad y>a .
$$

*o.abdullaev@mathinst.ru, https://orcid.org/0000-0001-8503-1268

(c) Siberian Federal University. All rights reserved 
There are few works where local and non-local problems for the parabolic-hyperbolic type equation with Caputo operator and loaded terms were studied (see [11, 12] and references therein). Similar problems for the loaded parabolic-hyperbolic type equations that include several integrodifferential operators of fractional order such as Riemann-Liouville, Erdelyi-Kober or, among others, Atangana-Baleano operators were considered (see [13-15]). We would like to note that the equations in the above mentioned woks have only linear loaded terms.

The main goal of this work is to prove the existence and uniqueness of solution of an analogue of the Gellerstedt problem with non-local assumptions on the boundary and integral gluing conditions for equation (1).

Let $\Omega^{+}$be a bounded domain with segments $A_{1} A_{2}=\{(x, y): x=1,0<y<h\}, B_{1} B_{2}=$ $=\{(x, y): x=0,0<y<h\}, B_{2} A_{2}=\{(x, y): y=h, 0<x<1\}$ at $y>0 . \Omega_{1}=\left\{A_{1} C_{1} E\right\}$ and $\Omega_{2}=\left\{B_{1} C_{2} E\right\}$ are characteristic triangles bounded with characteristics $A_{1} C_{1}: x-y=1$, $E C_{1}: x+y=l$ and $B_{1} C_{2}: x+y=0, E C_{2}: x-y=l,(0<l<1)$, respectively, of equation (1) at $y<0$, where $A_{1}(1 ; 0), A_{2}(1 ; h), B_{1}(0 ; 0), B_{2}(0 ; h), C_{1}\left(\frac{l+1}{2} ; \frac{l-1}{2}\right), C_{2}\left(\frac{l}{2} ; \frac{-l}{2}\right), E(l, 0)$.

The following designations are used: $\Omega=\Omega^{+} \cup \Omega_{1} \cup \Omega_{2} \cup\left(A_{1} B_{1}\right), I=\{y: 0<y<h\}$, $I_{1}=\{x: 0<x<l\}, I_{2}=\left\{x: l<x<\frac{l+1}{2}\right\}, I_{3}=\{x: 0<x<1\}$.

The following two problems are considered in the domain $\Omega$ :

Problem I. To find a solution $u(x, y)$ of equation 1 in the following class of functions:

$$
W=\left\{u(x, y): u(x, y) \in C(\bar{\Omega}) \cap C^{2}\left(\Omega_{1} \cup \Omega_{2}\right) \quad u_{x x} \in C\left(\Omega^{+}\right),{ }_{C} D_{o y}^{\alpha} u \in C\left(\Omega^{+}\right)\right\} .
$$

The solution satisfies boundary conditions

$$
\begin{gathered}
\left.u(x, y)\right|_{A_{1} A_{2}}=\varphi_{1}(y),\left.\quad u(x, y)\right|_{B_{1} B_{2}}=\varphi_{2}(y), \quad 0 \leqslant y \leqslant h, \\
\left.u(x, y)\right|_{E C_{1}}=\psi_{1}(x), l \leqslant x \leqslant \frac{l+1}{2}, \\
\left.u(x, y)\right|_{B_{1} C_{2}}=\psi_{2}(x), 0 \leqslant x \leqslant \frac{l}{2},
\end{gathered}
$$

and gluing condition

$$
\begin{aligned}
\lim _{y \rightarrow+0} y^{1-\alpha} u_{y}(x, y)= & \lambda_{1}(x) u_{y}(x,-0)+\lambda_{2}(x) u_{x}(x,-0)+ \\
& +\lambda_{3}(x) \int_{0}^{x} r(t) u(t, 0) d t+\lambda_{4}(x) u(x, 0)+\lambda_{5}(x), \quad 0<x<1,
\end{aligned}
$$

where $\varphi_{j}(y), \psi_{j}(x)(j=1,2), \lambda_{k}(x)(k=\overline{1,5})$ are given functions such that $\sum_{k=1}^{4} \lambda_{k}^{2}(x) \neq 0$. The required class of functions is specified later.

Problem II. To determine a solution $u(x, y)$ of equation (1) in the class of functions $W$ that satisfies all conditions of Problem I except condition (5) which is replaced by

$$
\frac{d}{d x} u\left(\frac{x}{2}, \frac{-x}{2}\right)=a_{1}(x) u_{y}(x, 0)+a_{2}(x) u_{x}(x, 0)+a_{3}(x) u(x, 0)+a_{4}(x), \quad 0<x<l,
$$

where $a_{k}(x)(k=\overline{1,4})$ are given functions such that $\sum_{k=1}^{3} a_{k}^{2}(x) \neq 0$.

Condition (7) is called the non-local condition which connect linear combination of values of functions $u_{y}(x, 0), u_{x}(x, 0)$ and $u(x, 0)$ at the points of the interval $B_{1} E$ with the value of $\frac{d}{d x} u\left(\frac{x}{2}, \frac{-x}{2}\right)$ at the points of the characteristic $B_{1} C_{2}$. 


\section{Main functional relations}

It is well-known that solution of the Cauchy problem for equation

$$
u_{x x}-u_{y y}+f_{2}(x, y ; u(x, 0))=0, \text { at } y<0
$$

with initial conditions $u(x, 0)=\tau(x), 0 \leqslant x \leqslant 1 ; u_{y}(x,-0)=\nu^{-}(x), \quad 0<x<1$ can be represented as follows:

$$
u(x, y)=\frac{\tau(x+y)+\tau(x-y)}{2}-\frac{1}{2} \int_{x+y}^{x-y} \nu^{-}(t) d t-\frac{1}{4} \int_{x+y}^{x-y} d \eta \int_{x+y}^{\eta} f_{2}\left(\frac{\xi+\eta}{2}, \frac{\xi-\eta}{2} ; \tau\left(\frac{\xi+\eta}{2}\right)\right) d \xi
$$

If we set $a_{1}(x)=a_{2}(x)=a_{3}(x)=0$ and $a_{4}(x)=\psi_{2}^{\prime}(x)$ then Problem $\mathrm{I}$ is a special case of Problem II. Then we will study the existence and uniqueness of solutions of Problem II.

Using condition (7) and relation (8), we obtain

$$
\begin{aligned}
\left(2 a_{1}(x)+1\right) \nu^{-}(x)=\frac{1}{2} \int_{0}^{x} f_{2} & \left(\frac{\xi+x}{2}, \frac{\xi-x}{2} ; \tau\left(\frac{\xi+x}{2}\right)\right) d \xi+ \\
& +\left(1-2 a_{2}(x)\right) \tau^{\prime}(x)-2 a_{3}(x) \tau(x)-2 a_{4}(x), 0<x \leqslant l .
\end{aligned}
$$

Similarly, using condition (4) and relation (8), we obtain

$$
\nu^{-}(x)=\tau^{\prime}(x)-\frac{1}{2} \int_{l}^{x} f_{2}\left(\frac{\xi+x}{2}, \frac{\xi-x}{2} ; \tau\left(\frac{\xi+x}{2}\right)\right) d \xi-\psi_{1}^{\prime}\left(\frac{l+x}{2}\right), \quad l \leqslant x<1
$$

Let $\lim _{y \rightarrow+0} y^{1-\alpha} u_{y}(x, y)=\nu^{+}(x)$. Using gluing condition (6), we have

$$
\nu^{+}(x)=\lambda_{1}(x) \nu^{-}(x)+\lambda_{2}(x) \tau^{\prime}(x)+\lambda_{3}(x) \int_{0}^{x} r(t) \tau(t) d t+\lambda_{4}(x) \tau(x)+\lambda_{5}(x), \quad 0<x<1 .
$$

On the other hand, taking into account (11) and to $\lim _{y \rightarrow 0} D_{0 y}^{\alpha-1} f(y)=\Gamma(\alpha) \lim _{y \rightarrow 0} y^{1-\alpha} f(y)$, we obtain from equation (1) at $y \rightarrow+0$ that

$$
\begin{aligned}
\tau^{\prime \prime}(x)-\Gamma(\alpha) \lambda_{1}(x) \nu^{-}(x) & -\Gamma(\alpha) \lambda_{2}(x) \tau^{\prime}(x)-\Gamma(\alpha) \lambda_{3}(x) \int_{0}^{x} r(t) \tau(t) d t- \\
& -\Gamma(\alpha) \lambda_{4}(x) \tau(x)+f_{1}(x, 0 ; \tau(x))-\Gamma(\alpha) \lambda_{5}(x)=0, \quad 0<x<1 .
\end{aligned}
$$

\section{Uniqueness of solution of Problem II}

Assuming $\lambda_{5}(x) \equiv 0$, we multiply equation (12) by $\tau(x)$ and then integrate it from 0 to 1 :

$$
\begin{aligned}
& \int_{0}^{1} \tau^{\prime \prime}(x) \tau(x) d x-\Gamma(\alpha) \int_{0}^{1} \lambda_{2}(x) \tau(x) \tau^{\prime}(x) d x-\Gamma(\alpha) \int_{0}^{1} \lambda_{3}(x) \tau(x)\left(\int_{0}^{x} r(t) \tau(t) d t\right) d x- \\
& \quad-\Gamma(\alpha) \int_{0}^{1} \lambda_{4}(x) \tau^{2}(x) d x-\Gamma(\alpha) \int_{0}^{1} \lambda_{1}(x) \tau(x) \nu^{-}(x) d x+\int_{0}^{1} \tau(x) f_{1}(x, 0 ; \tau(x)) d x=0 .
\end{aligned}
$$

Obviously, if $\tau(0)=\tau(1)=0$ then, integrating by parts, we obtain

$$
\int_{0}^{1} \tau^{\prime \prime}(x) \tau(x) d x=-\int_{0}^{1} \tau^{\prime 2}(x) d x \leqslant 0
$$




$$
\Gamma(\alpha) \int_{0}^{1} \lambda_{2}(x) \tau(x) \tau^{\prime}(x) d x=-\frac{\Gamma(\alpha)}{2} \int_{0}^{1} \lambda^{\prime}{ }_{2}(x) \tau^{2}(x) d x .
$$

Taking into account that

$$
\begin{gathered}
2 \Gamma(\alpha) \int_{0}^{1} \lambda_{3}(x) \tau(x)\left(\int_{0}^{x} r(t) \tau(t) d t\right) d x=\Gamma(\alpha) \int_{0}^{1} \frac{\lambda_{3}(x)}{r(x)} d\left(\int_{0}^{x} r(t) \tau(t) d t\right)^{2}= \\
=\Gamma(\alpha) \frac{\lambda_{3}(1)}{r(1)}\left(\int_{0}^{1} r(t) \tau(t) d t\right)^{2}-\Gamma(\alpha) \int_{0}^{1}\left(\frac{\lambda_{3}(x)}{r(x)}\right)^{\prime}\left(\int_{0}^{x} r(t) \tau(t) d t\right)^{2} d x
\end{gathered}
$$

we obtain that inequality

$$
\Gamma(\alpha) \int_{0}^{1} \lambda_{3}(x) \tau(x)\left(\int_{0}^{x} r(t) \tau(t) d t\right) d x \geqslant 0
$$

is satisfied provided that

$$
\frac{\lambda_{3}(1)}{r(1)} \geqslant 0, \text { and }\left(\frac{\lambda_{3}(x)}{r(x)}\right)^{\prime} \leqslant 0 .
$$

Let us consider now the integral

$$
\begin{gathered}
J=\Gamma(\alpha) \int_{0}^{1} \lambda_{1}(x) \tau(x) \nu^{-}(x) d x-\int_{0}^{1} \tau(x) f_{1}(x, 0 ; \tau(x)) d x= \\
=\Gamma(\alpha) \int_{0}^{l} \lambda_{1}(x) \tau(x) \nu^{-}(x) d x+\Gamma(\alpha) \int_{l}^{1} \lambda_{1}(x) \tau(x) \nu^{-}(x) d x-\int_{0}^{1} \tau(x) f_{1}(x, 0 ; \tau(x)) d x .
\end{gathered}
$$

Taking (9) and (10) into account when $\psi_{1}(x) \equiv a_{4}(x) \equiv 0$ and assuming $1+2 a_{1}(x) \neq 0$, we obtain

$$
\begin{array}{r}
J=-\Gamma(\alpha) \int_{0}^{l} \tau(x) A_{1}(x) d x \int_{0}^{x} f_{2}\left(\frac{\xi+x}{2}, \frac{\xi-x}{2} ; \tau\left(\frac{\xi+x}{2}\right)\right) d \xi+\Gamma(\alpha) \int_{0}^{l} B(x) \tau(x) \tau^{\prime}(x) d x- \\
-\int_{0}^{1} \tau(x) f_{1}(x, 0 ; \tau(x)) d x-\Gamma(\alpha) \int_{0}^{l} C(x) \tau^{2}(x) d x+\Gamma(\alpha) \int_{l}^{1} \lambda_{1}(x) \tau(x) \tau^{\prime}(x) d x- \\
-\Gamma(\alpha) \int_{l}^{1} \tau(x) A_{2}(x) d x \int_{l}^{x} f_{2}\left(\frac{\xi+x}{2}, \frac{\xi-x}{2} ; \tau\left(\frac{\xi+x}{2}\right)\right) d \xi,
\end{array}
$$

where $A_{i}(x)=\frac{(-1)^{i} \lambda_{1}(x)}{2\left(1+2 a_{1}(x)\right)^{2-i}}(i=1,2), B(x)=\frac{1-2 a_{2}(x)}{1+2 a_{1}(x)} \lambda_{1}(x), C(x)=\frac{2 a_{3}(x) \lambda_{1}(x)}{1+2 a_{1}(x)}$.

Thus, assuming that

$$
\begin{gathered}
\Gamma(\alpha) \int_{0}^{l} B(x) \tau(x) \tau^{\prime}(x) d x=\frac{\Gamma(\alpha)}{2} \int_{0}^{l} B(x) d\left(\tau^{2}(x)\right)=-\frac{\Gamma(\alpha)}{2} \int_{0}^{l} \tau^{2}(x) B^{\prime}(x) d x, \\
\Gamma(\alpha) \int_{l}^{1} \lambda_{1}(x) \tau(x) \tau^{\prime}(x) d x=\frac{\Gamma(\alpha)}{2} \int_{l}^{1} \lambda_{1}(x) d\left(\tau^{2}(x)\right)=-\frac{\Gamma(\alpha)}{2} \int_{l}^{1} \tau^{2}(x) \lambda_{1}^{\prime}(x) d x
\end{gathered}
$$

and using (16), we have

$$
J=\Gamma(\alpha) \int_{0}^{1} \lambda_{1}(x) \tau(x) \nu^{-}(x) d x-\int_{0}^{1} \tau(x) f_{1}(x, 0 ; \tau(x)) d x \geqslant 0
$$

if $A_{i}(x) \tau(x) f_{2}(s, s-x ; \tau(s)) \leqslant 0, \quad \tau(x) f_{1}(x, 0 ; \tau(x)) \leqslant 0, \quad B^{\prime}(x) \leqslant 0, \quad \lambda_{1}^{\prime}(x) \leqslant 0$ and $C(x) \leqslant 0$. 
Thus, considering (14), (15), (16), (17), (18) and assuming that $\frac{1}{2} \lambda_{2}^{\prime}(x)-\lambda_{4}(x) \leqslant 0$, it follows from (13) that $\tau(x) \equiv 0$.

Hence, based on the solution of the first boundary problem (1) and (3) we obtain $u(x, y) \equiv 0$ in $\bar{\Omega}^{+}$. Further, taking into account that $\tau(x) \equiv 0$, we obtain from functional relations (9) and (10) that $\nu^{-}(x) \equiv 0$. Consequently, taking into account solution (8), we have $u(x, y) \equiv 0$ in closed domain $\bar{\Omega}_{j}(j=1,2)$.

Let us assume that $f_{i}(x, y ; \tau(x))=0$ at $\tau(x)=0$. Then the following theorem can be formulated

Theorem 2.1. Let us assume that conditions

$$
\begin{gathered}
A_{i}(x) \geqslant 0(\leqslant 0), \quad(i=1,2), \quad \tau(x) f_{2}(s, s-x ; \tau(s)) \geqslant 0(\leqslant 0) ; \\
\tau(x) f_{1}(x, 0 ; \tau(x)) \leqslant 0, \quad B^{\prime}(x) \leqslant 0, \quad \lambda_{1}^{\prime}(x) \leqslant 0, \quad C(x) \leqslant 0 ; \\
\frac{\lambda_{3}(1)}{r(1)} \geqslant 0, \quad\left(\frac{\lambda_{3}(x)}{r(x)}\right)^{\prime} \leqslant 0, \quad \frac{1}{2} \lambda_{2}^{\prime}(x)-\lambda_{4}(x) \leqslant 0,
\end{gathered}
$$

are valid then the solution $u(x, y)$ of Problem II is unique if it exists.

\section{Existence of solution of Problem I}

Theorem 3.1. If conditions (19), (20), (21) and

$$
\begin{gathered}
f_{1}(x, y ; \tau(x)) \in C\left(\overline{\Omega^{+}}\right) \cap C^{1}\left(\Omega^{+}\right), \quad f_{2}(x, y ; \tau(x)) \in C\left(\overline{\Omega_{1}} \cup \overline{\Omega_{2}}\right) \cap C^{1}\left(\Omega_{1} \cup \Omega_{2}\right) ; \\
\left|f_{j}\left(x, y ; \tau_{2}(x)\right)-f_{j}\left(x, y ; \tau_{2}(x)\right)\right| \leqslant L_{j}\left|\tau_{2}(x)-\tau_{1}(x)\right|, \quad L_{j}=\text { const }>0(j=1,2) ; \\
\varphi_{1}(y), \varphi_{2}(y) \in C(\bar{I}) \cap C^{1}(I), \quad \psi_{i}(x) \in C\left(\overline{I_{2}}\right) \cap C^{2}\left(I_{2}\right) ; \\
a_{i}(x) \in C^{1}\left(\overline{I_{1}}\right) \cap C^{2}\left(I_{1}\right), \quad \lambda_{k}(x) \in C^{1}\left(\overline{I_{3}}\right) \cap C^{2}\left(I_{3}\right), \quad i=\overline{1,4}, \quad k=\overline{1,5}
\end{gathered}
$$

are fulfilled then there exists a solution of Problem I.

Proof. Taking (9) and (10) into account, from (12) we obtain that

$$
\begin{gathered}
\tau^{\prime \prime}(x)=F_{1}(x), \quad 0<x<l, \\
\tau^{\prime \prime}(x)=F_{2}(x), \quad l<x<1,
\end{gathered}
$$

where

$$
\begin{array}{r}
F_{1}(x)=\Gamma(\alpha) \lambda_{3}(x) \int_{0}^{x} r(t) \tau(t) d t+\Gamma(\alpha) A_{1}(x) \int_{0}^{x} f_{2}\left(\frac{\xi+x}{2}, \frac{\xi-x}{2} ; \tau\left(\frac{\xi+x}{2}\right)\right) d \xi- \\
-f_{1}(x, 0 ; \tau(x))+\Gamma(\alpha)\left(B(x)+\lambda_{2}(x)\right) \tau^{\prime}(x)-\Gamma(\alpha)\left(C(x)-\lambda_{4}(x)\right) \tau(x)+D(x), \\
\begin{array}{r}
F_{2}(x)=\Gamma(\alpha) \lambda_{3}(x) \int_{l}^{x} r(t) \tau(t) d t-\Gamma(\alpha) A_{2}(x) \int_{l}^{x} f_{2}\left(\frac{\xi+x}{2}, \frac{\xi-x}{2} ; \tau\left(\frac{\xi+x}{2}\right)\right) d \xi- \\
-f_{1}(x, 0 ; \tau(x))+\Gamma(\alpha)\left(\lambda_{1}(x)+\lambda_{2}(x)\right) \tau^{\prime}(x)+\Gamma(\alpha) \lambda_{4}(x) \tau(x)+ \\
+\Gamma(\alpha)\left(\lambda_{5}(x)-\lambda_{1}(x) \psi_{1}^{\prime}\left(\frac{l+x}{2}\right)\right)
\end{array}
\end{array}
$$


and $D(x)=\Gamma(\alpha)\left(\lambda_{5}(x)-\frac{2 \lambda_{1}(x) a_{4}(x)}{1+2 a_{1}(x)}\right)$.

Solutions of equations (26) and (27) together with conditions

$$
\tau(0)=\varphi_{2}(0), \quad \tau(l)=\psi_{1}(l) \text { and }, \quad \tau(l)=\psi_{1}(l), \quad \tau(1)=\varphi_{1}(0),
$$

respectively, are

and

$$
\tau(x)=\int_{0}^{x}(x-t) F_{1}(t) d t-\frac{x}{l} \int_{0}^{l}(l-t) F_{1}(t) d t+\left(1-\frac{x}{l}\right) \varphi_{2}(0)+\frac{x}{l} \psi_{1}(l)
$$

$$
\tau(x)=\int_{l}^{x}(x-t) F_{2}(t) d t+\frac{l-x}{1-l} \int_{l}^{1}(1-t) F_{2}(t) d t+\frac{1-x}{1-l} \psi_{1}(l)+\frac{x-l}{1-l} \varphi_{1}(0) .
$$

Further, substituting (28) and (29) into (30) and (31), respectively, we obtain

$$
\begin{gathered}
\tau(x)=\Gamma(\alpha) \int_{0}^{x} r(z) \tau(z) d z \int_{z}^{x}(x-t) \lambda_{3}(t) d t-\frac{\Gamma(\alpha)}{l} x \int_{0}^{l} r(z) \tau(z) d z \int_{z}^{l}(l-t) \lambda_{3}(t) d t- \\
-\Gamma(\alpha) \int_{0}^{x}\left[(x-t)\left(B(t)+\lambda_{2}(t)\right)\right]^{\prime} \tau(t) d t-\Gamma(\alpha) \int_{0}^{x}(x-t)\left(C(t)-\lambda_{4}(t)\right) \tau(t) d t+ \\
\quad+\frac{\Gamma(\alpha) x}{l} \int_{0}^{l}\left[(l-t)\left(B(t)+\lambda_{2}(t)\right)\right]^{\prime} \tau(t) d t+ \\
+\frac{\Gamma(\alpha) x}{l} \int_{0}^{l}(l-t)\left(C(t)-\lambda_{4}(t)\right) \tau(t) d t+F_{1}^{*}(x)+\Phi_{1}(x, \tau(x)), \quad 0 \leqslant x \leqslant l
\end{gathered}
$$

where

$$
\begin{gathered}
F_{1}^{*}(x)=\int_{0}^{x}(x-t) D(t) d t-\frac{x}{l} \int_{0}^{l}(l-t) D(t) d t+\left(1-\frac{x}{l}\right) \varphi_{2}(0)+\frac{x}{l} \psi_{1}(l) \\
\Phi_{1}(x, \tau(x))=\frac{x}{l} \int_{0}^{l}(l-t) f_{1}(t, 0 ; \tau(t)) d t+ \\
\quad+\frac{\Gamma(\alpha) x}{l} \int_{0}^{l}(l-t) A_{1}(t) d t \int_{0}^{t} f_{2}\left(\frac{\xi+t}{2}, \frac{\xi-t}{2} ; \tau\left(\frac{\xi+t}{2}\right)\right) d \xi- \\
-\int_{0}^{x}(x-t) f_{1}(t, 0 ; \tau(t)) d t-\Gamma(\alpha) \int_{0}^{x}(x-t) A_{1}(t) d t \int_{0}^{t} f_{2}\left(\frac{\xi+t}{2}, \frac{\xi-t}{2} ; \tau\left(\frac{\xi+t}{2}\right)\right) d \xi
\end{gathered}
$$

and

$$
\begin{gathered}
\tau(x)=\Gamma(\alpha) \int_{l}^{x} r(z) \tau(z) d z \int_{z}^{x}(x-t) \lambda_{3}(t) d t+\frac{\Gamma(\alpha)(l-x)}{1-l} \int_{l}^{1} r(z) \tau(z) d z \int_{z}^{l}(l-t) \lambda_{3}(t) d t- \\
-\Gamma(\alpha) \int_{l}^{x}\left[(x-t)\left(\lambda_{1}(t)+\lambda_{2}(t)\right)\right]^{\prime} \tau(t) d t+\Gamma(\alpha) \int_{l}^{x}(x-t) \lambda_{4}(t) \tau(t) d t+ \\
\begin{array}{r}
\frac{\Gamma(\alpha)(l-x)}{1-l} \int_{l}^{1} \lambda_{4}(t) \tau(t) d t-\frac{\Gamma(\alpha)(l-x)}{1-l} \int_{l}^{x}\left[(1-t)\left(\lambda_{1}(t)+\lambda_{2}(t)\right)\right]^{\prime} \tau(t) d t+ \\
+F_{2}^{*}(x)+\Phi_{2}(x, \tau(x)), \quad l \leqslant x \leqslant 1,
\end{array}
\end{gathered}
$$

where

$$
\begin{aligned}
F_{2}^{*}(x) & =\Gamma(\alpha) \int_{0}^{x}(1-t)\left(\lambda_{5}(t)-\lambda_{1}(t) \psi_{1}^{\prime}\left(\frac{l+t}{2}\right)\right) d t- \\
& -\frac{l-x}{l} \int_{l}^{1}\left(\lambda_{5}(t)-\lambda_{1}(t) \psi_{1}^{\prime}\left(\frac{l+t}{2}\right)\right) d t+\frac{1-x}{1-l} \psi_{1}(l)+\frac{x-1}{1-l} \varphi_{1}(0)
\end{aligned}
$$


and

$$
\begin{gathered}
\Phi_{2}(x, \tau(x))=\frac{(x-l)}{1-l} \int_{l}^{1}(l-t) f_{1}(t, 0 ; \tau(t)) d t+ \\
+\frac{\Gamma(\alpha)(x-l)}{1-l} \int_{l}^{1}(l-t) A_{2}(t) d t \int_{l}^{t} f_{2}\left(\frac{\xi+t}{2}, \frac{\xi-t}{2} ; \tau\left(\frac{\xi+t}{2}\right)\right) d \xi- \\
-\int_{l}^{x}(x-t) f_{1}(t, 0 ; \tau(t)) d t-\Gamma(\alpha) \int_{l}^{x}(x-t) A_{2}(t) d t \int_{l}^{t} f_{2}\left(\frac{\xi+t}{2}, \frac{\xi-t}{2} ; \tau\left(\frac{\xi+t}{2}\right)\right) d \xi
\end{gathered}
$$

After some simplifications, equations (32) and (33) can be rewritten in the form of Fredholm integral equations of the second kind

$$
\tau(x)=\int_{0}^{l} K_{1}(x, z) \tau(z) d z+\widetilde{F}_{1}(x, \tau(x)), \quad 0 \leqslant x \leqslant l
$$

and

$$
\tau(x)=\int_{l}^{1} K_{2}(x, z) \tau(z) d z+\widetilde{F}_{2}(x, \tau(x)), \quad l \leqslant x \leqslant 1 .
$$

Here $\widetilde{F}_{j}(x, \tau(x))=F_{j}^{*}(x)+\Phi_{j}(x, \tau(x))(j=1,2)$, and

$$
K_{1}(x, z)=\left\{\begin{array}{c}
K_{11}(x, z) ; \quad 0 \leqslant z \leqslant x, \\
K_{12}(x, z) ; \quad x \leqslant z \leqslant l
\end{array} ; \quad K_{2}(x, z)=\left\{\begin{array}{l}
K_{21}(x, z) ; \quad l \leqslant z \leqslant x \\
K_{22}(x, z) ; x \leqslant z \leqslant 1
\end{array}\right.\right.
$$

with

$$
\begin{gathered}
K_{11}(x, z)=\Gamma(\alpha) r(z) \int_{z}^{x}(x-t) \lambda_{3}(t) d t-\frac{\Gamma(\alpha)}{l} x r(z) \int_{z}^{l}(l-t) \lambda_{3}(t) d t+ \\
+\Gamma(\alpha) \frac{l-x}{l}\left(B(z)+\lambda_{2}(z)\right)+\Gamma(\alpha) z \frac{l-x}{l}\left(B(z)+\lambda_{2}(z)\right)^{\prime}+\Gamma(\alpha) \frac{z(l-x)}{l}\left(C(z)-\lambda_{4}(z)\right), \\
K_{12}(x, z)=-\Gamma(\alpha) \frac{x}{l} r(z) \int_{z}^{l}(l-t) \lambda_{3}(t) d t+\frac{\Gamma(\alpha)}{l} x\left[(l-z)\left(B(z)+\lambda_{2}(z)\right)\right]^{\prime}+ \\
+\Gamma(\alpha) \frac{x}{l}(l-z)\left(C(z)-\lambda_{4}(z)\right), \\
K_{21}(x, z)=\Gamma(\alpha) r(z) \int_{z}^{x}(x-t) \lambda_{3}(t) d t+\frac{\Gamma(\alpha)(l-x)}{1-l} r(z) \int_{z}^{l}(l-t) \lambda_{3}(t) d t+ \\
+\Gamma(\alpha) \frac{l-x}{1-l}\left(\lambda_{1}(z)+\lambda_{2}(z)\right)-\Gamma(\alpha)(z-l) \frac{x-1}{1-l}\left(\lambda_{1}(z)+\lambda_{2}(z)\right)^{\prime}+\Gamma(\alpha) \frac{(z-l)(x-1)}{1-l} \lambda_{4}(z),
\end{gathered}
$$

and

$$
\begin{gathered}
K_{22}(x, z)=\Gamma(\alpha) \frac{l-x}{1-l} r(z) \int_{z}^{l}(l-t) \lambda_{3}(t) d t+\frac{\Gamma(\alpha)(x-l)}{1-l}\left[(1-z)\left(\lambda_{1}(z)+\lambda_{2}(z)\right)\right]^{\prime}+ \\
+\Gamma(\alpha) \frac{l-x}{1-l}(l-z) \lambda_{4}(z) .
\end{gathered}
$$

Besides, due to regularity of functions in (22), (23), (24) and (25) it is not difficult to verify that $\left|K_{j}(x, t)\right|$ and $\left|\widetilde{F}_{j}(x)\right|, \quad(j=1,2)$ are bounded. Moreover,

$$
\begin{gathered}
K_{1}(x, t) \in C([0, l] \times[0, l]) \cup C_{x, t}^{2,0}((0, l) \times(0, l)), \\
K_{2}(x, t) \in C([l, 1] \times[l, 1]) \cup C_{x, t}^{2,0}((l, 1) \times(l, 1))
\end{gathered}
$$


and $\widetilde{F}_{1}(x) \in C[0, l] \cup C^{2}(0, l), \quad \widetilde{F}_{2}(x) \in C[l, 1] \cup C^{2}(l, 1)$.

Since kernels $K_{j}(x, t)$ are continuous and functions $\widetilde{F}_{j}(x)$ are continuously differentiable, solutions of integral equations (34) and (35) can be derived in terms of resolvent-kernel as follows

$$
\tau(x)=\int_{0}^{l} R_{1}(x, z) \widetilde{F}_{1}(z, \tau(z)) d z+\widetilde{F}_{1}(x, \tau(x)), 0 \leqslant x \leqslant l
$$

and

$$
\tau(x)=\int_{l}^{1} \widetilde{F}_{2}(z, \tau(z)) R_{2}(x, z) d z+\widetilde{F}_{2}(x, \tau(x)), \quad l \leqslant x \leqslant 1,
$$

where $R_{j}(x, z)$ is the resolvent kernel of $K_{j}(x, z)$.

Considering functions $\Phi_{j}(x, \tau(x))(j=1,2)$ from (36) and (37), the Fredholm type nonlinear integral equations are constructed

$$
\begin{gathered}
\tau(x)=\int_{0}^{l} L_{12}(x, t) d t \int_{0}^{t} f_{2}\left(\frac{\xi+t}{2}, \frac{\xi-t}{2} ; \tau\left(\frac{\xi+t}{2}\right)\right) d \xi+ \\
+\int_{0}^{l} R_{1}(x, t) d t \int_{0}^{l} L_{12}(t, z) d z \int_{0}^{z} f_{2}\left(\frac{\xi+z}{2}, \frac{\xi-z}{2} ; \tau\left(\frac{\xi+z}{2}\right)\right) d \xi+ \\
+\int_{0}^{l} L_{11}(x, t) f_{1}(t, 0 ; \tau(t)) d t+\int_{0}^{l} R_{1}(x, t) d t \int_{0}^{l} L_{11}(t, z) d z f_{1}(z, 0 ; \tau(z)) d z+ \\
+F_{1}^{*}(x)+\int_{0}^{l} F_{1}^{*}(t) R_{1}(x, t) d t, \quad 0 \leqslant x \leqslant l
\end{gathered}
$$

and

where

$$
\begin{gathered}
\tau(x)=\int_{l}^{1} L_{22}(x, t) d t \int_{l}^{t} f_{2}\left(\frac{\xi+t}{2}, \frac{\xi-t}{2} ; \tau\left(\frac{\xi+t}{2}\right)\right) d \xi+ \\
+\int_{l}^{1} R_{2}(x, t) d t \int_{l}^{1} L_{22}(t, z) d z \int_{l}^{z} f_{2}\left(\frac{\xi+z}{2}, \frac{\xi-z}{2} ; \tau\left(\frac{\xi+z}{2}\right)\right) d \xi+ \\
+\int_{l}^{1} L_{21}(x, t) f_{1}(t, 0 ; \tau(t)) d t+\int_{l}^{1} R_{2}(x, t) d t \int_{l}^{1} L_{21}(t, z) f_{1}(z, 0 ; \tau(z)) d z+ \\
+F_{2}^{*}(x)+\int_{l}^{1} F_{2}^{*}(t) R_{2}(x, t) d t, \quad l \leqslant x \leqslant 1
\end{gathered}
$$

It is not difficult to verify that

$$
\begin{gathered}
L_{11}(x, t)=\left\{\begin{array}{ll}
\frac{t(l-x)}{l} ; & 0 \leqslant t \leqslant x, \\
\frac{x(l-t)}{l} ; & x \leqslant t \leqslant l
\end{array} ; \quad L_{12}(x, t)=\Gamma(\alpha) A_{1}(t) L_{11}(x, t) ;\right. \\
L_{21}(x, t)=\left\{\begin{array}{ll}
\frac{(t-l)(1-x)}{1-l} ; & l \leqslant t \leqslant x, \\
\frac{(1-t)(x-l)}{1-l} ; & x \leqslant t \leqslant 1
\end{array} ; L_{22}(x, t)=\Gamma(\alpha) A_{2}(t) L_{21}(x, t) .\right.
\end{gathered}
$$

$$
\left|\int_{0}^{l} L_{11}(x, t) d t\right| \leqslant \frac{2 l^{2}}{27}=M_{11} ;\left|\int_{l}^{1} L_{21}(x, t) d t\right| \leqslant \frac{2(1-l)^{2}}{27}=M_{21} .
$$

Now, assuming that $\left|\Gamma(\alpha) A_{j}(x)\right| \leqslant \beta_{j} ; \quad\left|\int_{0}^{1} R_{j}(x, t) d t\right| \leqslant \delta_{j}, \quad(j=1,2)$ and taking into account (40), we obtain

$$
\left|\int_{0}^{l} L_{12}(x, t) d t\right| \leqslant \beta_{1} M_{11}=M_{12} ; \quad\left|\int_{l}^{1} L_{22}(x, t) d t\right| \leqslant \beta_{2} M_{21}=M_{22},
$$




$$
\left|\int_{0}^{l} R_{1}(x, t) d t \int_{0}^{l} L_{1 j}(x, z) d z\right| \leqslant \delta_{1} M_{1 j}=N_{1 j},
$$

and

$$
\left|\int_{l}^{1} R_{2}(x, t) d t \int_{l}^{1} L_{2 j}(x, z) d z\right| \leqslant \delta_{2} M_{2 j}=N_{2 j},(j=1,2) .
$$

Solvability of integral equation (38) can be established with the use of the method of successive approximations. Let us assume $\tau_{0}(x)=\widetilde{F_{1}^{*}}(x)$ and define the functional sequence $\left\{\tau_{n}(x)\right\}$ in the following form:

$$
\begin{gathered}
\tau_{n}(x)=\int_{0}^{l} L_{12}(x, t) d t \int_{0}^{t} f_{2}\left(\frac{\xi+t}{2}, \frac{\xi-t}{2} ; \tau_{n-1}\left(\frac{\xi+t}{2}\right)\right) d \xi+ \\
+\int_{0}^{l} R_{1}(x, t) d t \int_{0}^{l} L_{12}(t, z) d z \int_{0}^{z} f_{2}\left(\frac{\xi+z}{2}, \frac{\xi-z}{2} ; \tau_{n-1}\left(\frac{\xi+z}{2}\right)\right) d \xi+ \\
+\int_{0}^{l} L_{11}(x, t) f_{1}\left(t, 0 ; \tau_{n-1}(t)\right) d t+\int_{0}^{l} R_{1}(x, t) d t \int_{0}^{l} L_{11}(t, z) f_{1}\left(z, 0 ; \tau_{n-1}(z)\right) d z+ \\
+F_{1}^{*}(x)+\int_{0}^{l} F_{1}^{*}(t) R_{1}(x, t) d t, \quad 0 \leqslant x \leqslant l
\end{gathered}
$$

where $\widetilde{F_{1}^{*}}(x)=F_{1}^{*}(x)+\int_{0}^{l} F_{1}^{*}(t) R_{1}(x, t) d t$.

Let us assume that

$$
\left|f_{1}(z, 0, \tau(z))\right| \leqslant m_{1},\left|f_{2}\left(\frac{\xi+z}{2}, \frac{\xi-z}{2}, \tau\left(\frac{\xi+z}{2}\right)\right)\right| \leqslant m_{2}, \quad\left(m_{1}, m_{2}>0\right) .
$$

Considering (22), (23) and taking into account (40)-(43), we obtain from (44) the following inequalities

$$
\begin{gathered}
\left|\tau_{1}(x)-\tau_{0}(x)\right| \leqslant M\left(m_{1}+m_{2}\right) \text {, where } M=\max \left\{M_{11}+N_{11} ; M_{12}+N_{12}\right\}, \\
\left|\tau_{2}(x)-\tau_{1}(x)\right| \leqslant\left|\tau_{1}(x)-\tau_{0}(x)\right| \cdot\left|L_{1}\left(M_{11}+N_{11}\right)+L_{2}\left(M_{12}+N_{12}\right)\right| \leqslant \\
\leqslant M\left(L_{1}+L_{2}\right)\left|\tau_{1}(x)-\tau_{0}(x)\right| \leqslant M^{2}\left(L_{1}+L_{2}\right)\left(m_{1}+m_{2}\right), \\
\left|\tau_{n}(x)-\tau_{n-1}(x)\right| \leqslant M\left(L_{1}+L_{2}\right)\left|\tau_{n-1}(x)-\tau_{n-2}(x)\right| \leqslant M^{n}\left(L_{1}+L_{2}\right)^{n-1}\left(m_{1}+m_{2}\right) .
\end{gathered}
$$

Thus, we have contraction mapping. Let us note that solvability of the considered problem was reduced to integral equations (34) and (35). Based on the uniqueness of solution of the problem and due to equivalence of the problem to integral equations in the sense of solvability, we establish that integral equations (34) and (35) have a unique solution. Since, an integral equation does not have more than one solution and we have contraction mapping, one can conclude that functional sequence $\left\{\tau_{n}(x)\right\}$ has a unique limiting function $\tau(x)$.

With the arguments given above one can prove solvability of equation (37). Unknown function $\nu^{-}(x)$ can be found from (9). Solution of Problem II in the domain $\Omega^{+}$is the solution of the first BVP [2,16]. Solution of Problem II in the domain $\Omega_{j}$ is given in (8). Hence, Theorem 3.1 is proved.

Remark. Let us note that functions $f_{i}(x, y, u(x, 0))=u^{p_{i}}(x, 0)$ satisfy our assumptions and all conditions on functions $f_{i}(x, y ; u(x, 0))$ at $p_{i}=$ const $>0(i=1,2)$. 


\section{References}

[1] A.A.Kilbas., O.A.Repin, An analog of the Tricomi problem for a mixed type equation with a partial fractional derivative, Fractional Calculus and Applied Analysis, 13(2010), no. 1, $69-84$.

[2] A.S.Berdyshev, E.T.Karimov, N.Akhtayeva, Boundary Value problems with integral gluing conditions for fractional order mixed type equations, Hindawi. IJDE, (2011).

[3] B.J.Kadirkulov, Boundary problems for mixed parabolic-hyperbolic equations with two lines of changing type and fractional derivative, EJDE, $\mathbf{5 7}(2014), 1-7$.

[4] A.M.Nakhushev, The loaded equations and their applications., Moscow, Nauka, 2012 (in Russian)

[5] A.M.Nakhushev, The Darboux problem for a certain degenerate second order loaded integral-differential equation, Differential equations, 12(1976), no. 1, 103-108.

[6] K.B.Sabitov, Initial-boundary problem for parabolic-hyperbolic equation with loaded summands, Russian Math. Iz. VUZ, 59(2015), no. 6, 23-33. DOI: 10.3103/S1066369X15060055

[7] E.P.Melisheva, Dirichlet problem for loaded equation of Lavrentiev-Bizadze, Vestnik SamGU. Estestvenno Nauchnaya Ser, (80)(2010), no. 6, 39-47 (in Russian).

[8] O.Kh.Abdullaev, A non-local problem for a loaded mixed-type equation with a integral operators, J. Samara State Tech. Univ., Ser. Phys. and Math. Sci., 20(2016), no.2, 220-240 (in Russian). DOI: 10.14498/vsgtu1485

[9] O.Kh.Abdullaev, K.Sadarangani, Non-local problems with integral gluing condition for loaded mixed type equations involving the Caputo fractional derivative, Electronic Journal of Differential Equations (EJDE), 164(2016), 1-10.

[10] A.A.Kilbas, H.M.Srivastava, J.J.Trujillo, Theory and Applications of Fractional Differential Equations, In: North-Holland Mathematics Studies, Vol. 204, Elsevier Science B.V., Amsterdam, 2006.

[11] M.S.Salakhitdinov, E.T.Karimov, On a nonlocal problem with gluing condition of integral form for parabolic-hyperbolic equation with Caputo operator, Reports of the Academy of Sciences of the Republic of Uzbekistan. (DAN RUz), (2014), no. 4, 69-79 (in Russian).

[12] A.S.Berdyshev, A.Cabada, E.T.Karimov, On a non-local boundary problem for a parabolichyperbolic equation involving a Riemann-Liouville fractional differential operator, Nonlinear Anal., Theory Methods Appl, 75(6)(2012), 3268-3273. DOI: 10.1016/j.na.2011.12.033

[13] K.Sadarangani, K.O.Kh.Abdullaev, A non-local problem with discontinuous matching condition for loaded mixed type equation involving the Caputo fractional derivative, Advances in Difference Equations, 2016, AIDE-D-16-00217R3. DOI: 10.1186/s13662-016-0969-1

[14] O.Kh.Abdullaev, Some Problems for the Degenerate Mixed Type Equation Involving Caputo and Atangana-Baleanu Operators Fractional Order, Progr. Fract. Differ. Appl., 6(2020), no. $2,101-114$. DOI: $10.18576 / \mathrm{pfda} / 060203$ 
[15] O.Kh.Abdullaev, About a problem for the degenerate mixed type equation involving Caputo and Erdelyi-Kober operators fractional order, Ukr. math. jour, 71(2019), no. 6. 723-738.

DOI: $10.1007 / \mathrm{s} 11253-019-01682-\mathrm{z}$

[16] A.V.Pskhu, Partial differential equation of fractional order, Moscow, Nauka, 2000 (in Russian).

\section{Разрешимость краевых задач для параболо-гиперболичес- кого уравнения с нелинейной нагруженной слагаемой \\ Обиджон Абдуллаев}

Институт математики им. В. И. Романовского Академии наук Республики Узбекистан Ташкент, Узбекистан Национальный университет Узбекистана Ташкент, Узбекистан

\footnotetext{
Аннотация. Данная работа посвящена доказательству существования и единственности краевой задачи с нелокальными краевыми и интегральными условиями склеивания для парабологиперболического уравнения с дробной производной Капуто. Применением метода интегралов энергии доказана единственность решения задачи. Существование решения было доказано методом интегральных уравнений.

Ключевые слова: дробная производная Капуто, нагруженное уравнение, интегральное условие склеивания, нелинейное интегральное уравнение, нелокальная задача, существование и единственность решения.
} 